\title{
The USE of Fuzzy Logic IN Automatic Control Systems
}

\author{
Oleg Veselov, Alexandr Veselov \& Stanislav Svetushenko
}
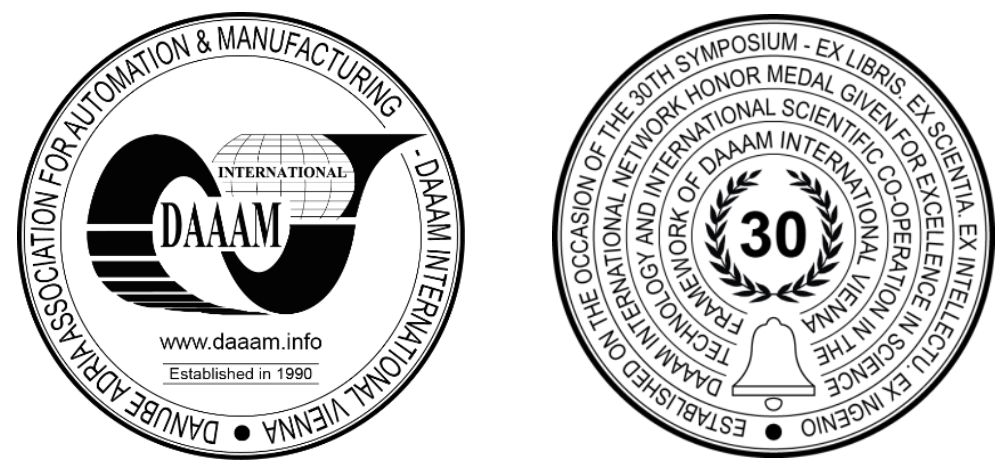

This Publication has to be referred as: Veselov, O[leg]; Veselov, A[lexandr] \& Svetushenko, S[tanislav] (2019). The Use of Fuzzy Logic in Automatic Control Systems, Proceedings of the 30th DAAAM International Symposium, pp.08970903, B. Katalinic (Ed.), Published by DAAAM International, ISBN 978-3-902734-22-8, ISSN 1726-9679, Vienna, Austria

DOI: $10.2507 / 30$ th.daaam.proceedings.125

\begin{abstract}
The paper deals with application of fuzzy controllers in automatic control systems. The use of fuzzy logic in automatic control systems (ACS) is specific, associated with essential features of organizational nature, in implementation of structures and operating algorithms. Intellectual systems of industrial automation in their development encounter certain difficulties: unsettled terminology, lack of conventional classification, immature methodology for designing fuzzy automatic control systems. The article formulates the definition of artificial intelligence and intelligent fuzzy ACS, based on the conventional terminology used in the theory of control and artificial intelligence. Classification of structures of automatic control systems is submitted according to the degree of introduction of fuzzy controllers (FC) in automatic control system. Block diagrams and their description are presented. The specific structure of FC, based on classical principles, is explained. It is emphasized that adaptive ACS is implemented using adaptive FC. The method of designing fuzzy controllers for ACS implementation is considered.
\end{abstract}

Keywords: Fuzzy logic; artificial intelligence; automatic control system; structure; fuzzy controller.

\section{Introduction}

One of the key directions on the way of creation of future productions, robots, numerical control machines in mechanical engineering prompted scientific and engineering society to use methods of artificial intelligence in implementation of automatic control systems.

On the one hand, traditional methods of system design do not lead to satisfactory results as the initial description of the problem to be solved is incomplete. The desire to obtain complete information for accurate mathematical modeling of the system leads to the waste of time and money, due to the structure complexity of real control systems.

In 1965 Zade published a fundamental work on the theory of fuzzy sets, in which he outlined its mathematical apparatus. In 1973 he presented the theory of fuzzy logic, and later he delivered the theory of soft computing and the theory of computing with words and perceptions.

This was followed by the development of a new direction in solving many problems in various fields of knowledge. At the same time it was the beginning of the use of fuzzy logic theory in implementation of technical systems. 
This statement is based on Wang's proof of the theorem (in 1992) that there is a fuzzy system that forms the output function, that the upper limit of the difference between the original function and the output is less than or equal to some arbitrary constant. In 1993 B. Cosco proved FAT (Fuzzy Approximation Theorem), the essence of which is that any mathematical system can be approximated by a system based on fuzzy logic. Finally, in 1995, Castro showed that the logical controller of Mamdani under certain conditions is a universal approximator. These scientific works facilitated active application of fuzzy logic in automatic control systems.

Application of the theory of fuzzy sets in the design of automatic control systems is explained by the fact that fuzzy systems are designed faster, they are obtained easier than clear analogues. Expert knowledge is easily introduced into fuzzy systems that enable to quickly create a system with operating algorithms understandable to an engineer. The training methods developed in the last decade allow to set up a fuzzy system to ensure the required quality of functioning [1], ..., [4].

The use of fuzzy logic in automatic control systems is specific, associated with the essential features of organizational nature, in implementation of structure and operating algorithms.

However, the further development of intelligent industrial automation systems faces certain difficulties. At present, there is the unsettled terminology regarding fuzzy logic in automatic control systems. The interpretation of certain points complicates understanding of the problem. Also a methodology for fuzzy ACS design has not been established. There is no clear definition of intelligent ACS, the level of their "intelligence", there is no generally accepted classification. There are a few publications in this field. At the same time, introduction of intelligent ACS is inevitable.

Nowadays one of the central concepts in control systems is the concept of artificial intelligence. There are many definitions of it [5], [6], [7], but there is no universal one more or less acceptable in technical systems. There are no methods determining the intelligence level of a particular control system.

Systems with artificial intelligence can have a large data storage, speed, ability to self-study and self-organization, they implement conclusions, make decisions, form expert assessments. They are a powerful tool in ACS design. It is especially important that such systems can operate in conditions of partial or complete uncertainty [8].

Fuzzy logic is also considered to be a part of artificial intelligence.

The aim is to increase operation efficiency of automatic control systems using artificial intelligence methods. To this effect it is necessary:

- to introduce terminology definitions;

- to design ACS with FC with a variable degree of implementation in the structure;

- to improve methodology of FC design for application in ACS.

\section{Basic concepts of intelligent ACS}

To assess the level of artificial intelligence of ACS it is necessary to define artificial intelligence, intelligent system and introduce the assessment scale of intelligence level.

Definition 1. Artificial intelligence is the collection and processing of information, the extraction of knowledge, its presentation, storing and using.

The terms used in the definition are generally understood, but the term "presentation" needs explanation [1].

Knowledge presentation is the most important area of artificial intelligence research. Knowledge can have different forms of object descriptions, for example, in the form of cause-and-effect or functional relations. Having adequate knowledge and ability to use it effectively means that the system is able to implement conclusions and make decisions to a certain extent. Usually neural networks are used to represent knowledge, fuzzy logic, soft computing, artificial neural networks, evolutionary computing, probabilistic reasoning, computing with words and perceptions. However, all methods of knowledge representation have some limitations related to the peculiarities of application field.

In this situation, to achieve this goal, it is necessary to find a way of expressing the rule specific to any system, including ACS. This is the essence of the problem of knowledge representation.

Therefore, the development of a theory or a method of knowledge representation of an arbitrary type is the important problem that will be relevant for a long time.

Definition 2. Intelligent system is a system consisting of an intelligent controller and a control object.

The important features of intelligent systems are: the ability to analyze and explain their actions and knowledge, the acquisition of new knowledge and changing it in accordance with their behavior, ensuring the relationship between the process and the goal of control. In technical terms, intelligent ACS should have a large data storage, high speed, ability to self-study and self-organization, to implement conclusions and make decisions.

In publications regarding fuzzy logic there are concepts of control system and automatic control system, and the concepts are mixed. This problem significantly complicates understanding of the task. In this regard, we will try to distinguish these concepts.

Definition 3. A control system is a complex of functionally interconnected and interacting parts that jointly perform a specific task and have properties which are not included in parts of the system separately and provide control of any object or process. The example of such systems is software control system.

Definition 4. Automatic control system is a complex of control objects and a controller, interacting in accordance with the control algorithm. Conventional terms applied in the theory of automatic control are used in the definitions. 


\section{Structures of fuzzy automatic control systems}

It follows from the above that it is necessary to comply the known principles while designing a fuzzy controller. At the same time, there is a specific application of fuzzy logic in ACS. In the majority of works a fuzzy controller (FC) is considered as a control system external to the controller. In other words, it is used to form an additional control algorithm. In a few works FC becomes an integral part of the control system and is used as a regulator in the direct channel. [10], [11,] [12], [13].

In this case, we consider FC as a universal tool that provides optimal characteristics of systems, adaptive or extreme controllers, and controllers with special types of motion, etc.

The conducted analysis made it possible to carry out systematization of the use of FC in ACS [8], [14], ..., [18].

The following definition of fuzzy automatic control systems is proposed according to the degree of FC introduction into ACS structure.

1. ACS with fuzzy controller. A control system in which a fuzzy controller producing a control action based on a fuzzy output is used as a regulator(s). Due to initialized loaded data in the form of a rule base, the fuzzy inference system performs certain functions and cannot be modified on its own (fig. 1a, b).

In a sense, it is a "hard" controller - inference rules, terms and data base, formed once, remain unchanged. The controller can be installed anywhere in the structure of ACS (in the direct channel (Fig. 1a), in the feedback loop (Fig. 1b)).

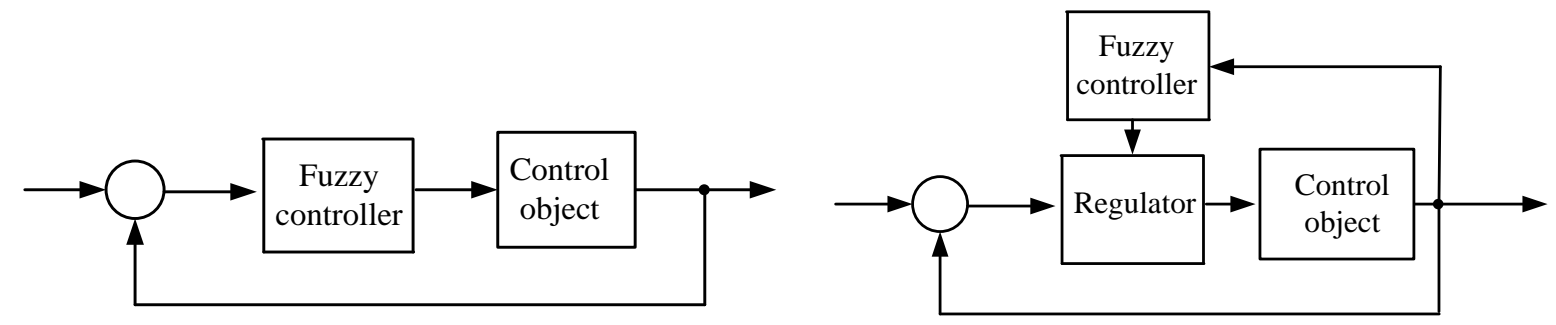

a)

b)

Fig. 1. ACS with fuzzy controller: a) FC in the direct channel, b) FC in the feedback loop

2. Interconnected fuzzy ACS. The control system in which the circuit uses a classic controller and fuzzy controller correcting parameters of the classical controller based on fuzzy output algorithms (Fig. 1b). In this case, the input variables are the state variables of the control object, and the output variables are the parameters of the control law. The system becomes more flexible to change the properties of the object and it is possible to modify the control action in accordance with the rules based on knowledge.

3. Hierarchical fuzzy ACS. The control system uses a fuzzy controller(s) as the controller(s) with the possibility of modifying the rule base using one of the fuzzy output methods formed by one of FC (Fig. 2).

When changing the operating environment, the upper level of the fuzzy ACS performs a reconfiguration of the fuzzy inference system for subsequent levels.

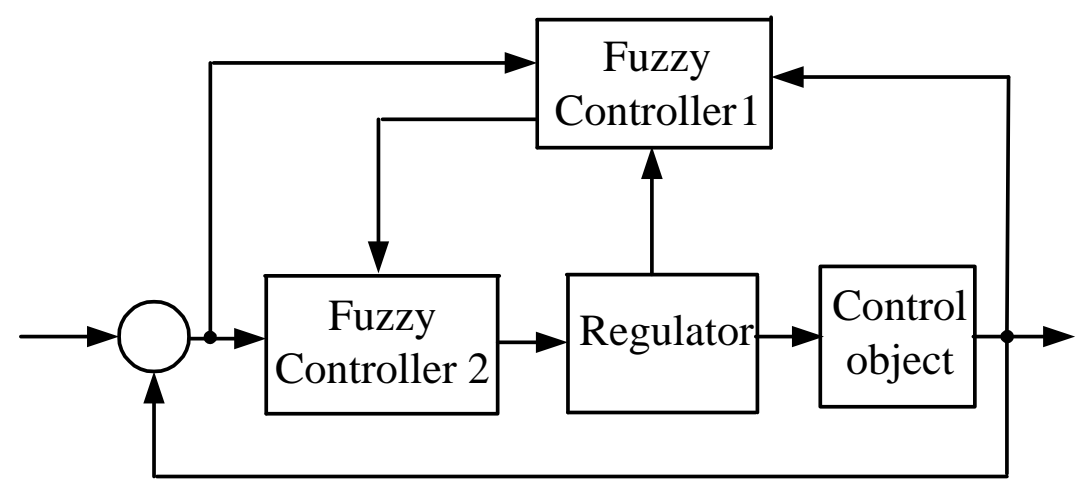

Fig. 2. Hierarchical fuzzy ACS

4. Hybrid fuzzy ACS. Neural networks and fuzzy controllers are used as a regulator(s), which use training or selfstudy of the neural network with implementation of conclusions and decision-making in a fuzzy controller, providing special types of motion, optimal, adaptive or other conditions for operation of ACS (Fig. 3). 


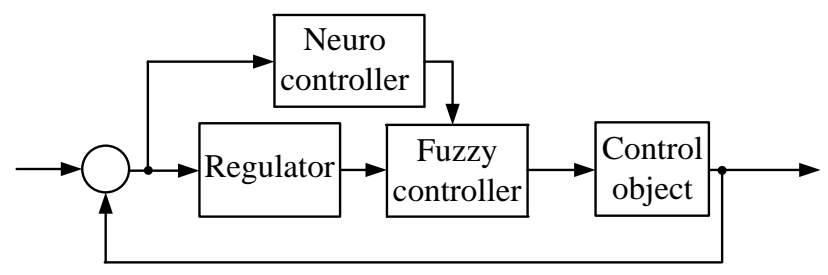

Fig. 3. Hybrid fuzzy ACS

5. Cascade fuzzy ACS in which controllers are switched on in sequence, in parallel or in sequence parallel or parallely in sequence.

6. Network fuzzy ACS containing a set of FC interconnected on the terms of cause-effect relations, in which the relationship provides the exchange of information so that each FC is able to share with each other, performing the operating task in any way (Fig. 4).

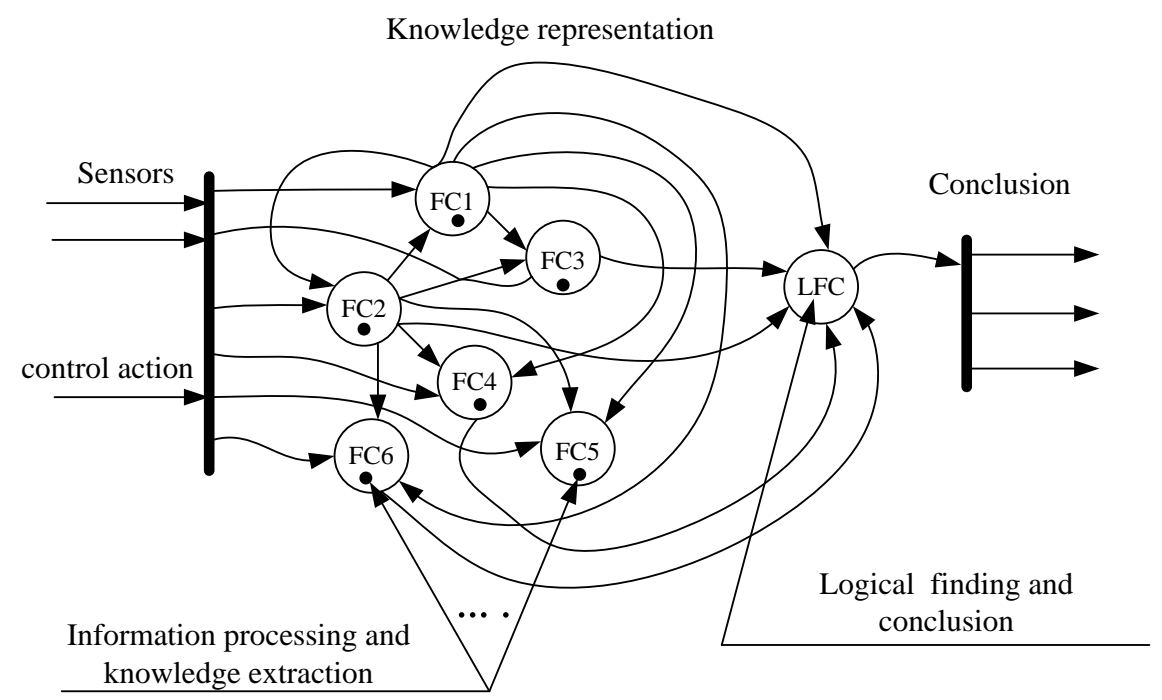

Fig. 4. Module structure of fuzzy network ACS

Particular conclusions in each FC are transmitted to logical finding and conclusion, where the final decision is made.

7. Combined fuzzy ACS - in such systems all of the above are used in various combinations.

8. Fuzzy ACS (full fuzzy) is an automatic control system, which contains no other solutions except fuzzy controllers, interconnected in any way, or it is a single controller with multiple inputs and outputs. In this case, FC should have the ability to self-organization and self-study, changing the type of functions of terms membership and intervals of input and output sets; (have the property of modification and adjust to the solution of the current task), change the data base wholly or in part, replenish or reduce it; to implement logical deduction and the method of defuzzification (Fig. 5).

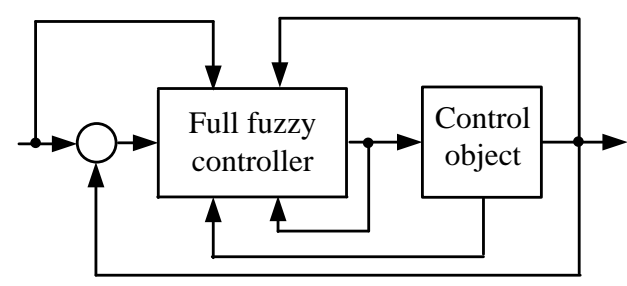

Fig. 5. The structure of a full fuzzy module

The principles of adaptive control are of particular interest. Typically, an adaptive system contains the scheme as a "core" implementing one of the fundamental principles of control, and the adaptation circuit is attached to it as a secondary one performing parameter correction. The adaptation circuit usually consists of a measuring device, calculating device and controlling device (Fig.6a).

Adaptation circuit can also be implemented in the form of FC (Fig. 6b) obviously having a measuring device, calculating device and controlling device (Fig. 6c). 
However, as well as ACS FC can be implemented as an adaptive device. Thus, adaptive ACS is realized on the basis of adaptive FC.

But in this case, the adaptive FC solves the problem of adaptation to changing input data by changing its parameters or the structure, or both, in order to achieve an optimal logical conclusion for implementation of adaptive control of ACS. At the same time, the FC can be installed in the direct path of the ACS (Fig. 6c) and perform the functions of an adaptive controller [2].
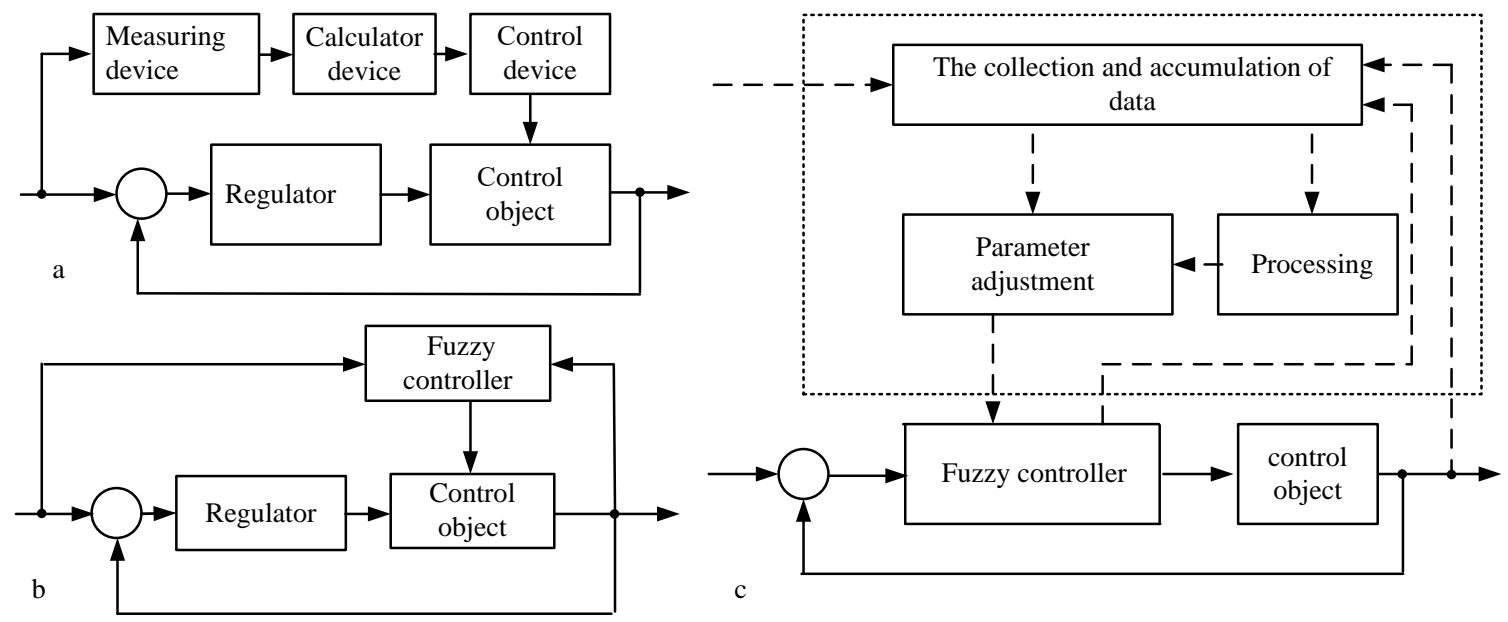

Fig. 6. Adaptive ACS structures: a) classical adaptive structure without feedback, b) adaptive structure with fuzzy controller, c) adaptive structure and adaptive controller in the direct channel

This classification of fuzzy systems is not final, because currently there are efforts to develop the intelligence of fuzzy ACS. These goals can be achieved by combining different approaches to design intelligent systems as part of hierarchical intelligent controllers, combining seamlessly principles and methods of fuzzy inference, situational control, knowledge engineering, neural networks and evolutionary modeling, using soft computing and big data.

\section{Method of creating fuzzy controllers for fuzzy automatic control systems}

Development of intelligent ACS is the most promising they are built on the basis of fuzzy neural networks, that allows to combine both methods of work with fuzzy information and knowledge, and the ability of systems to selfreconfiguration at the software and hardware level.

It is not possible to give general recommendations on the choice of solution - it depends on application and specifics of control object operation. However, it is possible to follow two ways of design: ascending conclusions, from symptoms to a conclusion, or top-down conclusions, from the actual result to symptoms [9].

For ACS, like any technical system using controllers with fuzzy logic, we can formulate the following steps [17], [19]:

- to set a goal of application and choose the structure of FC;

To choose the structure of FC according to the implemented output characteristics of the control process, operating conditions and control actions.

Being a universal approximator, FC is able to calculate any function that can be used in its setting. It is possible to recommend the following structures of FC, on the principle of design the structure and operation algorithm. The following structures are not exhaustive.

1. Classic FC. Once built it is not able to change its properties. It is easy to implement, provides satisfactory accuracy.

2. Adjustable FC. Parameter setting depends on the changing operating conditions. For example, it is possible to use spline activation functions, which can be deformed, adjusting to the changing parameters of the system or expand (narrow) the range of sets carriers. To change $\alpha$ - section of activation functions, etc.

3. Tunable FC - the change in the structure is implemented by introduction of feedback, changeover between FC, connecting additional sets, changing of transmission channels of conclusion results, etc.

4. Adaptive FC - in case of unforeseen changes in external signals in input or output, FC on the basis of analysis changes automatically its parameter points or its structure so as to preserve the intended quality of its operation.

5. A self-adjusting FC, in which a certain parameter has a domain of definition, depending on the operating conditions, is adjusted to ensure its stability. This can be achieved, for example, by changing the number of terms or the field of their definition. The parameter of FC, the most liable to instability, is chosen.

6. FC with a reference model implemented as a "hard" FC, in which the difference between the current value of output signal and the model value is compensated by changing the data base, reduce the output result to the smallest error, etc.

- organization of the database for controller training, determination of the terms number of input and output variables and conversion of actual data into linguistic variables (fuzzification). 
The form of the signal and the range of parameters change in the given points of the system are determined at different input signals and under various operating conditions, taking disturbances into account. In fact, the set of measured parameters involved in the implementation of FC is determined. Carriers of fuzzy sets are determined.

Since many situations in object control is not constant due to instability and unpredictability of the object properties and external influences, the use of analytical models is inexpedient. Preference is given to structural models of the real system for which FC is designed.

Organization of the database due to the complexity of the systems and limitations on the measurement of variables is the key prerogative of models.

Calling on experts' aid to collect data is appropriate, but the data would have a purely linguistic meaning and would need to be ranked according to the technical characteristics of the systems in order to be used in the real operating procedure. Experts' opinions are often controversial and require additional approvals, especially for systems with incomplete information on the structure.

- $\quad$ selection of the type of fuzzy variables membership functions.

In practice, any dependencies that approximate the input and output data with the expected accuracy can be used as membership functions. The number of terms in the interval representation is substantial, as well as specifics of superposition of membership functions, the ways of their setting, the final and initial look of the functions and specifically of the output.

- generation of the data base and communication between background space and conclusion space.

Knowledge base is the most essential component in the structure of FC. Data processing is based either on the rules or on fuzzy relations.

Linguistic rules are written in the block of generation of the logical decision on the basis of knowledge (base of rules). The corresponding formulation of the rules achieves a result in which at least one of the rules is acceptable for any linguistic value of the control action. It is noted that substitution of any rules on a set of other rules or their multiplication, allows to design a system of any degree of complexity, focused on operation problems solving.

Max-Min Inference method of logical solution is the most frequently used. The resulting membership function with the maximum validity value is selected by means of mutual superposition.

This process combines all the basic concepts of fuzzy set theory: membership functions, linguistic variables, fuzzy logical operations, methods of fuzzy implication and fuzzy composition.

It is possible to get a decisive rule (conclusion) using the well-known methods Sugeno, Mamdani, Larsen and Tsukamoto. If these methods do not meet your expectations, develop your original method of implementing the conclusion, but this is not an easy task. Care should be taken to choose the method of logic conclusion and composition rules.

- a method of converting linguistic variables into numerical ones (defuzzification).

There are several methods for converting linguistic variables into numeric variables that differ in accuracy. This is First-of-Maxima - the first maximum, Middle-of-Maxima - the average maximum, Max-Criterion - the maximum criterion, Height Defuzzification - the largest of all $\alpha$ - levels.

\section{Conclusion}

The introduction of fuzzy controllers in ACS is inevitable due to the wide possibilities of fuzzy logic and its applications, taking into account that microprocessors perform logical operations much faster than arithmetic, and fuzzy algorithms are designed much easier.

ACS structures with FC have been designed, the place and role of FC in ACS structure have been identified in accordance with the tasks to be solved, increasing operation efficiency due to ease of implementation as compared with clear analogues. Application of given definitions eliminates unconformity in terminology.

The given classification of fuzzy systems is not final, because currently there are efforts to develop the intelligence of fuzzy ACS. These goals can be achieved by combining different approaches to design intelligent systems as part of hierarchical intelligent controllers, combining seamlessly principles and methods of fuzzy inference, control systems, knowledge engineering, neural networks and evolutionary modeling, using soft computing and big data.

Therefore, the development of a theory or a method of knowledge representation of an arbitrary type is the important problem that will be relevant for a long time.

The assessment of the intelligence level of fuzzy ACS is very complicated. Obviously the decision is connected with expert assessments. To be sure, the structure of full fuzzy has the maximum of artificial intelligence and the structure with a simple fuzzy controller has the minimum.

Designing of the ACS structure with a fuzzy controller is a creative process and there is no ready-made formula. Modeling will help to solve the whole complex of problems in the design process.

In the future, the internal FC structures will be designed within the framework of the designed ACS structures. The articles, taking the results of the research adduced in this paper, are ready for publication.

\section{Acknowledgments}


Research is executed with financial support of the Russian Fund of basic researches in the scientific project №1808-01126 a.

\section{References}

[1] Fuzzy sets in control and artificial intelligence models (1986)/ Under the editorship of D.A. Pospelov.- M.: Science. CH. ed. Fiz.-Mat. lit., 1986. - 312 p.- (Problems of artificial intelligence).

[2] Applied fuzzy systems: translated from the Japanese. (1993)/ K. Asai, D. Watada, S. Iwai and others; edited by T. Terano, K. Asai, M. Sugeno. - M.: World, 1993. — IvSBN 5-03-002326-7.

[3] Emelyanov, S.V. (2014) Artificial intelligence and decision-making: Methods of reasoning and knowledge representation. Cognitive research. Intelligent system. Issue.3 / S. V. Emelyanov. - M.: Lenand, 2014. - 120p.

[4] Intelligent automatic control systems/ ed. of I. M. Makarov, V. M. Lochina — M: FIZMATLIT, 2001 — 576 p. ISBN 5-9221-0162-5.

[5] Ryazantsev, V.D. (comp.) (2011) Large Polytechnic dictionary. - Moscow: Peace and education, 2011. — 704p. — ISBN 978-594666-621-3.

[6] Vasiliev, V.I. (2012 ) Intellectual information security systems: Textbook / V.I. Vasiliev; Reviewer R.M. Asadullin [et al.]. - Moscow: Mechanical Engineering, 2012. - 171 p.

[7] Smart Enough Systems: How to Deliver Competitive Advantage by Automating Hidden Decisions, 1st Edition, ISBN 0132347962, by Taylor James; and Raden, Neil; published by Pearson Education, Inc, publishing as Prentice Hall, Copyright (C) 2007 by Pearson Education, Inc.

[8] Watanabe H. and Dettloff. (1988) Reconfigurable fuzzy logic processor: A full custom digital VLCI, in Int. WorkshoponFuzzySystemsApplications, Iiruka, Japan, Aug. 1988, pp. 49-50.

[9] Townsend K., Focht D. (1990) Design and software implementation of expert systems on personal computers: TRANS. with English. /Preface. G. S. Osipova.- Moscow: Finance and statistics, 1990.- 320 p.ISBN 5-279-0255-0.

[10] Yagashita O., Itoh O., and Sugeno M. (1985) Application of fuzzy reasoning to the water purification process, in Industrial Applications of Fuzzy Control, Sugeno M, Ed. Amsterdam: North-Holand 1985, pp.19-40.

[11] Yasunobu S., Miyamoto S., and Ihara H. (1983) Fuzzy control for automatic train operation system, in Proc. 4th. IFAC/IFIP/IFORS Int. Congress on Control in Transportation Systems, Baden-Baden, April, 1983.

[12] Antamoshin, A.N. (2016) Intelligent control systems organizational and technical systems / A.N. Antamoshin, O.V. Blinova, A.V. Beans and others - M.: GLT , 2016. - 160 p.

[13] Klos, S[viatoslav]; Fedoryshyn, R[oman]; Savytskyi, V[olodymyr]; Pistun, Y[evhen] \& Matiko, F[edir] (2017). Classification of Automatic Controllers Diagrams, Proceedings of the 28th DAAAM International Symposium, pp.0967-0973, B. Katalinic (Ed.), Published by DAAAM International, ISBN 978-3-902734- 11-2, ISSN 17269679, Vienna, Austria. DOI: 10.2507/28th.daaam.proceedings.134.

[14] Yasunobu S., and Hasegawa T. (1987) Predictive fuzzy control and its applications for automatic container crane operation system, in Proc. 2nd. IFSA Congress, Tokyo, Japan, Julie 1987.

[15] Evgenev, G.B. (2012) Intellectual design systems: Textbook /G.B. Evgeniev. - M.: MGTU im. Bauman, 2012. - 410 p.

[16] Markov, N. G. (2014) Intellectual navigation and telecommunication control systems of mobile objects using cloud computing technology / N. G. Markov, D. M. Sonkin, A. S. Fadeev, etc. - M.: Fig, 2014. - 158c.

[17] Sovetov, B.Ya. (2015) Intellectual systems and technologies: Textbook / B. Ya. Sovetov. - Moscow: Academy, 2015. -624 p.

[18] Mamdani E. H. (1974) Applications of fuzzy algorithms for simple dynamic plant. Porc. IEE. vol. 121, n. 12, pp. 1585-1588, 1974.

[19] Zade L.A. The concept of a linguistic variable and its application to approximate reasoning. Part 1, 2, 3 //Information Sciences, n. 8 pp.199-249, pp.301-357; n. 9 pp. 43-80. 